a uniform B field", quoting the B field inside a long solenoid as an example. They assume that $\partial \mathbf{B} / \partial t$ is constant, claiming that, according to Maxwell's equations, the "time-varying $\mathbf{B}$ field gives rise to an $\mathbf{E}$ field", and adding "we have a static $\mathbf{E}$ field yet one that is not generated by charges". As an alternative "physical" interpretation, it is a straightforward boundary value problem to show that, inside the solenoid, in cylindrical coordinates the vector potential is $\mathbf{A}_{\Theta}=\mu_{0} n I r / 2$; then, using

$\mathbf{B}=\nabla \times \mathbf{A}$ and $\mathbf{E}=(-\nabla \phi-\partial \mathbf{A} / \partial t)$ for quasi-stationary conditions we obtain $B_{\mathrm{z}}=\mu_{0} n I$ and $E_{\Theta}=-(1 / 2) \mu_{0} n r(\mathrm{~d} I / \mathrm{d} t)$. It is straightforward to show that $\nabla \times \mathbf{E}=-\partial \mathbf{B} / \partial t$ for these fields. Hence both $B$ and the induced electric field $\mathbf{E}$ can be determined independently from the vector potential and the equation $\nabla \times \mathbf{E}=-\partial \mathbf{B} / \partial t$ is just a relation between these fields. By integrating the relation $\nabla \times \mathbf{E}=-\partial \mathbf{B} / \partial t$ at a fixed time we can equate $\oint \mathbf{E}$.dl to the rate of change of magnetic flux, without relating $\mathbf{E}$ and $\mathbf{B}$ back to their common source, and without saying that the $\nabla \times \mathbf{E}$ produces the $-\partial \mathbf{B} / \partial t$ or vice versa.

When discussing the displacement current, Thomas and Meadows again adopt the old ether viewpoint, writing "a nonzero $\partial \mathbf{E} / \partial t$ gives rise to a spatially varying B"; yet, for example, the $\partial \mathbf{E} / \partial t$ term never appears as one of the sources of $\mathbf{A}$, and hence of $\mathbf{B}=\nabla \times \mathbf{A}$, in the expression for the retarded vector potential. Maxwell's equations are then used as the

\section{Astrometric angles}

\section{Derek McNally}

Spherical Astronomy. By Robin M. Green. Cambridge University Press: 1985. Pp.520. Hbk £40, \$69.50; pbk£15, \$27.95.

Ar A time when astrometry is assuming an increasingly prominent role in astronomical research, it is essential to have available a good, up-to-date undergraduate textbook on the subject. R.M. Green was responsible for the last (1977) revision of Smart's classical Spherical Astronomy, but while there is a kinship between that book and this new one published under the same title, the content has been changed entirely.

A feature of textbooks such as Smart's and my own Positional Astronomy of 1974 was the exclusion of mathematical techniques which would not be familiar to beginning students. Both Smart and myself also concentrated on providing explanation of simplified practices which would allow rapid determination of parameters of limited accuracy. However, considerable advances in astrometric precision have basis of brief but lucid accounts of electromagnetic waves in empty space and conductors, and there are also good discussions of the Poynting vector and radiation pressure. Overall, this book only gives a brief and simplified development of the physical principles of Maxwell's equations and their applications, but students may find it a useful companion to the more detailed accounts of the subject.

Students should do a large number of problems to develop their expertise in electromagnetism. To this end, C.M. Lerner's Problems and Solutions in Electromagnetic Theory contains 425 solved problems covering all levels from firstyear undergraduate to graduate; some problems are in cgs units, the others in SI units. The solutions are given in full, all the steps are explained clearly, and the book will be a very helpful complement to the standard textbooks on electromagnetism.

The central theme of Electromagnetic Wave Theory by J.R. Wait is the application of the impedance concept to wave phenomena. This approach has been particularly fruitful in ionospheric physics, antenna theory and geophysics, areas where Wait himself has made outstanding contributions. This is an advanced book which gives a comprehensive account of the methods concerned for the benefit of the more general reader at graduate level.

W.G.V. Rosser is a Reader in the Department of Physics, University of Exeter, Exeter EX $44 Q J$, UK.

been made in recent years, and more are likely in the next decade with the advent of the astrometric satellite HIPPARCOS and the Space Telescope. While a rigorous relativistic treatment has been given by Murray in Vectorial Astrometry (1983), there has remained a need for a book intermediate in level between Smart/ McNally and Murray. That need is well met by the present text.

The book has the advantage that it gives a simpler introduction than Murray to the relativistic basis for astrometric measurement. It also retains a derivation of the older, first-order formulae for day-to-day use. It is important, as it always was, that an astrometric textbook lays the foundation for a thorough development of the subject, while recognizing that only a few of its readers will ever want to use the full detail.

Dr Green develops his arguments vectorially, which is a concise and useful way of working and provides a direct link to Murray's monograph. He explains the recent changes in the form of dynamical time used in the Astronomical Almanac and also gives a short summary of the mechanics of precession as well as the more usual description of its effects. It is

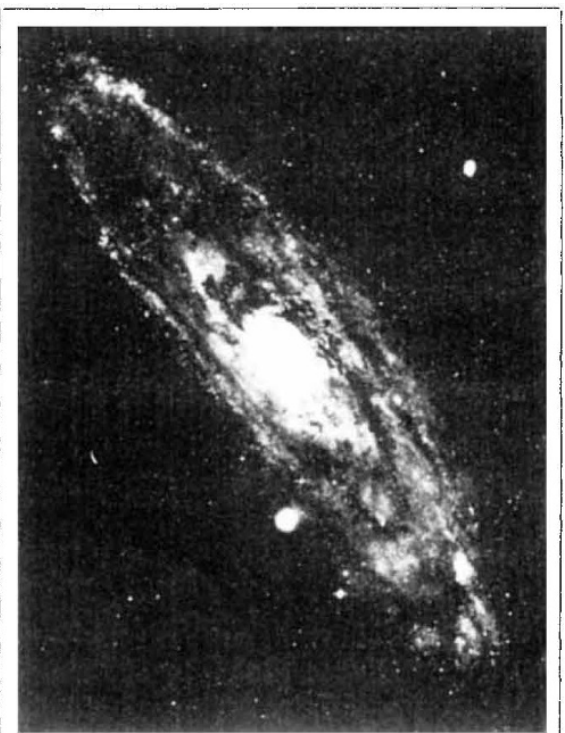

Andromeda Galaxy, photographed through the $0.9 \mathrm{~m}$ telescope of the Lick Observatory. The picture is taken from the paperback edition of The New Astronomy, by Nigel Henbest and Michael Marten, to be published next week by Cambridge University Press. Price is $£ 9.95, \$ 14.95$

pleasing to note that precession is treated here in terms of three rotations, rather than by the algebraically simpler method used by Smart. A very welcome new addition to a book at undergraduate level is an account of the astrometry of radioastronomy; this is a field where astrometric results of the highest precision are being obtained and it is likely to play a leading role in future work on fundamental reference systems.

There are a few niggles. For example, more use might have been made of vector methods. On the other hand, while a text of this sort is under continual tension between the need for clarity and the need for conciseness, the lack of an extended account of the calendar, seasons, rising, setting and twilights is regrettable. It is surprising, too, to see a discussion of dependences under plate measurement dependences are really only justified as a quick laboratory technique giving equatorial or rectangular equatorial co-ordinates directly.

Altogether, though, Dr Green has produced a good standard work, pitched at about second-year undergraduate level, which sets out the modern basis and terminology of astrometry. One hopes it will lead to a much better understanding, appreciation and use of astrometry by future generations of astronomers. Many of today's astronomers remain unaware of the contributions, actual and potential, of the subject - they now have little excuse for not ensuring that their successors are astrometrically aware.

Derek McNally is a Senior Lecturer in the Department of Physics and Astronomy, University College London, and Assistant Director of the University of London Observatory, Mill Hill Park, London NW7 2QS, UK. 